\title{
Overview of the experimental achievements in nucleon and meson polarizabilites
}

\author{
R. Miskimen* \\ University of Massachusetts, Amherst \\ E-mail: miskimen@physics.umass.edu
}

\begin{abstract}
An overview is presented of recent measurements of (i) proton and neutron scalar polarizabilities $\alpha_{E 1}$ and $\beta_{M 1}$, (ii) proton spin-polarizabilites $\gamma_{E 1 E 1}, \gamma_{M 1 M 1}, \gamma_{E 1 M 2}$, and $\gamma_{M 1 E 2}$, and (iii) charged and neutral pion scalar polarizabilities. The experiments completed, in progress, or in preparation are at Compass (CERN), HIGS, GlueX (JLab), Lund, and Mainz A2. These experiments span a range in beam energy from $100 \mathrm{MeV}$ to $190 \mathrm{GeV}$, and target mass number from $\mathrm{A}=1$ to 208
\end{abstract}

The 9th International workshop on Chiral Dynamics

17-21 September 2018

Durham, NC, USA

${ }^{*}$ Speaker. 


\section{Introduction}

The first measurement of the proton electromagnetic polarizability by Compton scattering (CS) with tagged photons took place at the University of Illinois $60 \mathrm{MeV}$ MUSL-2 microtron nearly 30 years ago [1]. Since then experimental and theoretical interest in polarizabilities has increased considerably. Polarizabilties are important test points for effective field theories, dispersion and lattice calculations, they test fundamental symmetries, and play a role in studies of proton radius and corrections to muon g-2. This CD2018 contribution covers recent experimental developments in the area of nucleon and meson polarizabilities. The experiments are presented in detail in the CD2018 contributions from E. Downie, M. Sikora, P. Martel, and D. Lawrence. For a comprehensive theoretical overview of the topic, see H. Gießhammer's contribution.

\section{Proton and neutron scalar polarizabilities}

The pivotal moment in this field was when the CS global analysis of McGovern et al. [2] was included in the PDG average. The current (2014) PDG values are:

$$
\alpha_{E 1}^{(p)}=(11.2 \pm 0.4) \times 10^{-4} \mathrm{fm}^{3}, \quad \beta_{M 1}^{(p)}=(2.5 \mp 0.4) \times 10^{-4} \mathrm{fm}^{3}
$$

While the uncertainty quoted in the PDG average is debatable, the PDG averages experimental results with the global analysis which includes those data, including a theoretical analysis of CS data in the PDG average was a big step forward.

The thrust of new proton polarizability measurements is to use linearly polarization asymmetry $\Sigma_{3}$ as an analyzer to measure $\alpha_{E 1}$ and $\beta_{M 1}$ separately, and independently of the Baldin sum rule value for $\alpha_{E 1}+\beta_{M 1}$. The linear polarization asymmetry, $\Sigma_{3}$, is defined as,

$$
\Sigma_{3}=\frac{d \sigma_{\|}-d \sigma_{\perp}}{d \sigma_{\|}+d \sigma_{\perp}}
$$

At Mainz a pilot measurement of $\Sigma_{3}$ showed that an extraction of $\alpha_{E 1}$ and $\beta_{M 1}$ from the linear polarization asymmetry is possible [3]. The sensitivity of the measurement to $\beta_{M 1}$ is approximately at the level of the PDG uncertainty in $\beta_{M 1}, \pm 0.4$ in the standard units. After the experiment took data, the Mainz photon tagger was upgraded to run at a rate approximately $\times 5$ higher rate than the old tagger focal plane. A new measurement of $\Sigma_{3}$ with the upgraded tagger focal plane obtained over 1 million good Compton scattering events over a range from $\mathrm{E}_{\gamma}=80$ to $140 \mathrm{MeV}$ and $\theta=$ $30^{\circ}$ to $135^{\circ}$, making this is the highest statistics Compton scattering data set ever obtained. Data analysis is in progress.

At HIGS CS data on the proton were recently taken with linearly polarized photons (see Mark Sikora's contribution to the proceedings). The photon beam at HIGS is exceptionally well suited for a LEX/ $\chi$ EFT theory based CS analysis for $\alpha_{E 1}$ and $\beta_{M 1}$; energies up to $100 \mathrm{MeV}$ are available, the beam energy is monochromatic with a spread of $\approx 4.5 \%$, photon intensities on the target are $\approx 10^{7}$ photons/sec, and the linear polarization is approximately $100 \%$.

Neutron scalar polarizabilities are obtained from the analysis of deuteron and helium CS data using accurate effective-field theory calculations. The favored approach is to use elastic scattering 
data, not quasi-free $\mathrm{d}\left(\gamma, \gamma^{\prime} \mathrm{n}\right) \mathrm{p}$ data, because (i) the elastic process is less complicated than the quasifree scattering and treatable through effective-field theory calculations, and (ii) elastic scattering has a larger cross section and greater sensitivity to the polarizabilities than the quasi-free process because of the Thompson term. The disadvantage of elastic scattering on deuteron is that it places a premium on the utilization of large $\mathrm{NaI}$ detectors with sufficient energy resolution to resolve elastic and inelastic scattering. Elastic scattering is also sensitive to the isoscalar polarizabilities, and therefore the proton contribution must be subtracted.

Data from Lund were recently published for elastic CS on the deuteron [4], nearly doubling the effective number of world data points and extending the energy range by $20 \mathrm{MeV}$ to higher energies. The experiment used the large-volume, segmented NaI(Tl) detectors BUNI, CATS, and DIANA to detect Compton-scattered photons. Because of unfavorable signal to background in the time-of-flight spectra, and the scattered photon energy distribution, this was a very challenging analysis. The results for the neutron are:

$$
\begin{aligned}
& \alpha_{E 1}^{(n)}=11.55 \pm 1.25(\text { stat }) \pm 0.2(B S R) \pm 0.8(\text { theory }) \times 10^{-4} \mathrm{fm}^{3} \\
& \beta_{M 1}^{(n)}=3.65 \mp 1.25(\text { stat }) \pm 0.2(B S R) \pm 0.8(\text { theory }) \times 10^{-4} \mathrm{fm}^{3}
\end{aligned}
$$

The compelling reason for doing CS on $Z>1$ nuclei is that the Thomson cross section goes as $Z^{2}$, with a better ratio of elastic to incoherent scattering compared to deuteron. There are plans at Mainz [5] to measure elastic CS on ${ }^{3} \mathrm{He}$ and ${ }^{4} \mathrm{He}$ with an active, gaseous helium target. The operating principle of the target is that ionizing particles produce UV scintillation light in the helium. The addition of a small amount of $\mathrm{N}_{2}$ shifts the scintillation light into the visible, and a SiPM photo-detector coupled to the target cell allows the He gas target to function as a detector. Elastic scattering can be separated from incoherent scattering by detecting the recoil helium nucleus in coincidence with the Compton-scattered photon. Development of the target is in progress, and test runs are anticipated in 2019.

\section{Proton spin-polarizabilities: experimental status}

Prior to the availability of single and double-polarized Compton scattering observables from the Mainz Compton scattering program, only two linear combinations of the polarizabilities were known. One combination is the forward spin polarizability:

$$
\gamma_{0}=-\gamma_{E 1 E 1}-\gamma_{E 1 M 2}-\gamma_{M 1 M 1}-\gamma_{M 1 E 2}=(-0.90 \pm 0.14) \times 10^{-4} \mathrm{fm}^{4}[6]
$$

which is fixed by the GTT sum rule, and the other combination is the backward spin polarizability:

$$
\gamma_{\pi}=-\gamma_{E 1 E 1}-\gamma_{E 1 M 2}+\gamma_{M 1 M 1}+\gamma_{M 1 E 2}=(8.0 \pm 1.8) \times 10^{-4} \mathrm{fm}^{4}[7]
$$

Here the standard convention is used of excluding the t-channel $\pi^{0}$-pole contribution from the spin-polarizabilities.

Single and double-polarized CS asymmetries in the $\Delta(1232)$ region have good sensitivity to the spin polarizabilities [8]. Measurements of this type provide essentially the only means by which the four lowest order spin polarizabilities $\gamma_{E 1 E 1}, \gamma_{M 1 M 1}, \gamma_{E 1 M 2}$ and $\gamma_{M 1 E 2}$ can be individually separated. 


\subsection{Compton scattering asymmetry with linearly polarized photons and unpolarized target}

The first measurements of $\Sigma_{3}$ in the $\Delta(1232)$ region were by the LEGS collaboration [9]. The LEGS experimental setup is typical of CS experiments in the $\Delta(1232)$ region, where the Compton photon is detected in a NaI detector, and the recoil proton is detected in a spectrometer arm specifically designed for recoil detection (LEGS), or in the same NaI detector used for Compton photon detection (Mainz Crystal Ball). Detecting the recoil proton is necessary to suppress background from $\pi^{0} \rightarrow \gamma \gamma$; the ratio of $\pi^{0}$ photoproduction to CS in the $\Delta(1232)$ region is approximately 100:1.

New data for the $\Sigma_{3}$ asymmetry in the $\Delta(1232)$ region have recently been taken by the Mainz A2 collaboration at the microtron MAMI using the Crystal Ball and TAPS detectors [10]. $\pi^{0}$ events are suppressed by making use of the hermeticity of the Crystal Ball, requiring that only one neutral and one charged track are present in the event. Additional background suppression is provided by imposing a co-planarity and an opening angle cut of $15^{\circ}$ on the direction of the recoil proton relative to the momentum transfer direction $\mathbf{q}$ defined by the incident and final photons. Figure 1 shows preliminary asymmetries from the analysis. The data has significant sensitivity to the $\gamma_{M 1 M 1}$ and $\gamma_{E 1 E 1}$ spin-polarizabilities. A letter on the result is in preparation for journal submission.

Fix $\gamma_{E 1 E 1}$

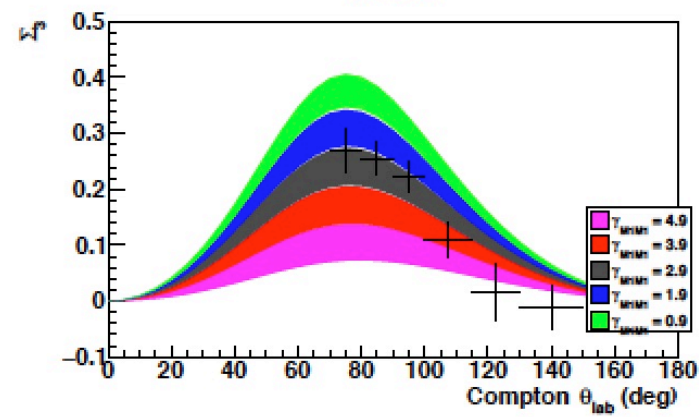

Fix $\gamma_{M 1 M 1}$

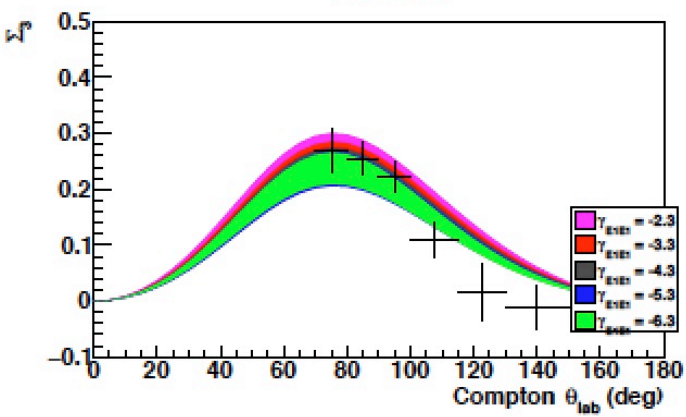

Figure 1: Preliminary linear polarization $\Sigma_{3}$ asymmetries in the $\Delta(1232)$ region[10]. The curves are from a dispersion theory calculation [8] with $\alpha_{E 1}, \beta_{M 1}, \gamma_{0}$, and $\gamma_{\pi}$ held fixed at their experimental values. The left plot has $\gamma_{E 1 E 1}$ fixed at -4.3 , and the colored bands are for $\gamma_{M 1 M 1}$ equal to 4.9, 3.9, 2.9, 1.9, and 0.9, in units of $10^{-4} \mathrm{fm}^{4}$. The width of each band represents the propagated errors from $\alpha_{E 1}, \beta_{M 1}, \gamma_{0}$, and $\gamma_{\pi}$ combined in quadrature. The right plot has $\gamma_{M 1 M 1}$ fixed at 2.9, and the colored bands are for $\gamma_{E 1 E 1}$ equal to $-2.3,-3.3$, $-4.3,-5.3$, and -6.3 .

\subsection{Compton scattering asymmetry with circularly polarized photons and transverse polarized target}

The relevant double-polarized CS asymmetry is defined as

$$
\Sigma_{2 x}=\frac{d \sigma_{x}^{R}-d \sigma_{x}^{L}}{d \sigma_{x}^{R}+d \sigma_{x}^{L}}
$$

where $d \sigma_{x}^{R(L)}$ is the differential cross section for transverse target polarization in the $x$-direction, and for right (left) circularly polarized photons. Data for the $\Sigma_{2 x}$ asymmetry in the $\Delta(1232)$ region have been published by the Mainz A2 collaboration [11]. Figure 2 shows the sensitivity of $\Sigma_{2 x}$ to 
the spin polarizabilities. The left figure shows little sensitivity to $\gamma_{M 1 M 1}$, and the right figure shows significant sensitivity to $\gamma_{E 1 E 1}$. Based on measurements of $\Sigma_{2 x}$ at angles $\theta \approx 90^{\circ}$, it is possible to uniquely identify $\gamma_{E 1 E 1}$. Martel et al. [11] performed the first global analysis of single and doublepolarized CS data in the $\Delta(1232)$ region to extract all four spin-polarizabilities.
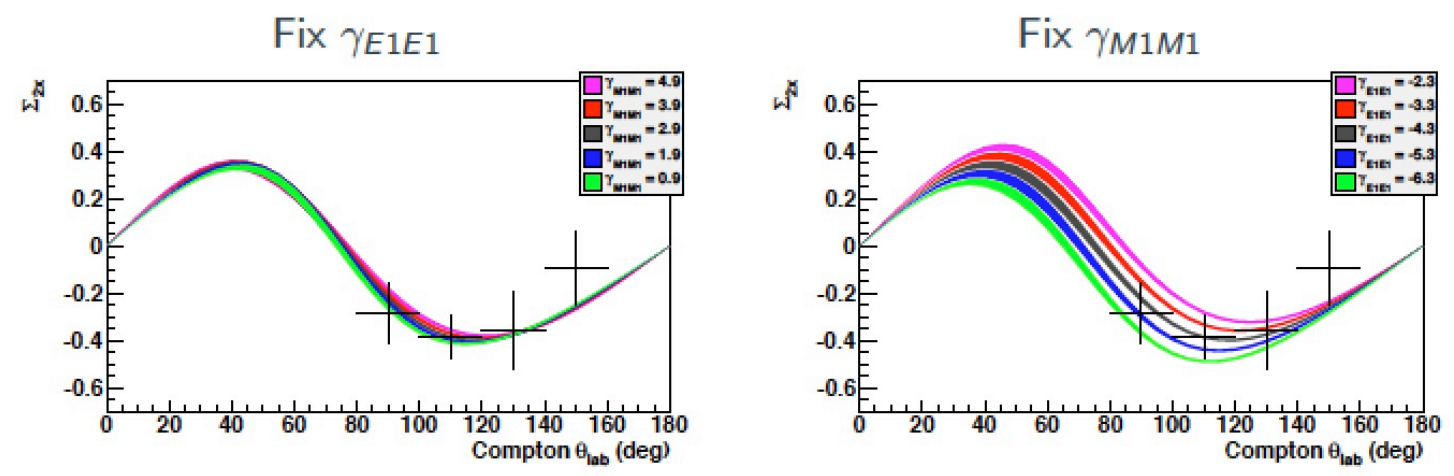

Figure 2: $\Sigma_{2 x}$ asymmetries in the $\Delta(1232)$ region[11]. The curves have the same meaning as in Figure 1.

\subsection{Compton scattering asymmetry with circularly polarized photons and longitudinal polarized target}

We finally consider the following double-polarized CS asymmetry with longitudinal target polarization,

$$
\Sigma_{2 z}=\frac{d \sigma_{z}^{R}-d \sigma_{z}^{L}}{d \sigma_{z}^{R}+d \sigma_{z}^{L}}
$$

where $d \sigma_{z}^{R(L)}$ is the differential cross section for right (left) circularly polarized photons to scatter from a nucleon target polarized in the incident beam direction. Note that the value of $\Sigma_{2 z}$ at the zero scattering angle is well-known from the sum rules for the forward CS amplitudes.

Figure 3 shows the sensitivity of $\Sigma_{2 z}$ to the spin polarizabilities. The left panel shows good sensitivity to $\gamma_{M 1 M 1}$, while the right panel shows less sensitivity to $\gamma_{E 1 E 1}$. The measurement of $\Sigma_{2 z}$ provides information on the spin-polarizabilities that is complementary to the information obtained from $\Sigma_{2 x}$. A letter on this result is being prepared for submission to PRL [14].

\subsection{Scintillating, spin-polarized target for low-energy Compton scattering}

Fig. 4 shows the proton detection efficiency as a function of proton kinetic energy for data taken with the Mainz frozen-spin target. The efficiency is derived from experimental $\pi^{0}$ photoproduction data. The drop in efficiency below $90 \mathrm{MeV}$ is caused by proton absorption in the frozen-spin target cryostat. This effectively limits incident Compton scattering energies to above $270 \mathrm{MeV}$ if recoil proton detection is required. The drop in efficiency above approximately 120 $\mathrm{MeV}$ is an artifact caused by the kinematic limit on proton kinetic energy.

Theorists prefer that polarized CS data be taken closer to the pion photo-production threshold, and within the range of validity for chiral effective field theory calculations. For this reason the Mainz polarized target group is developing an active polarized target using a polarizable scintillator. 

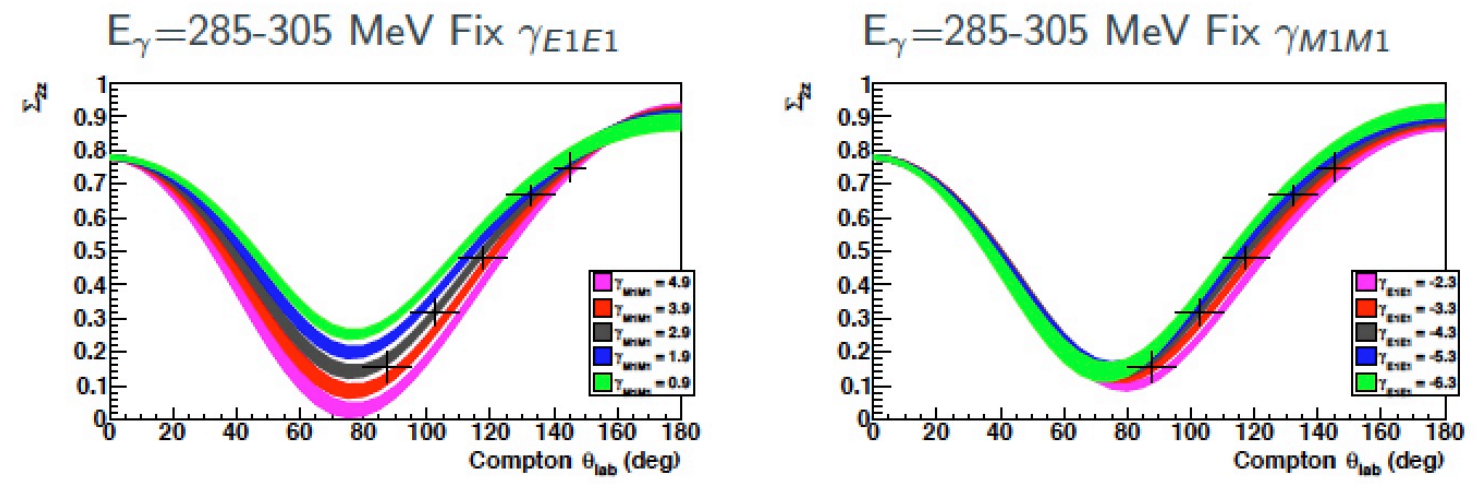

Figure 3: Preliminary $\Sigma_{2 z}$ asymmetries in the $\Delta(1232)$ region. The curves have the same meaning as in Figure 1.

There have been two test runs at Mainz with the active target, and for the second test run the scintillators were polarized. The data are currently under analysis [15].

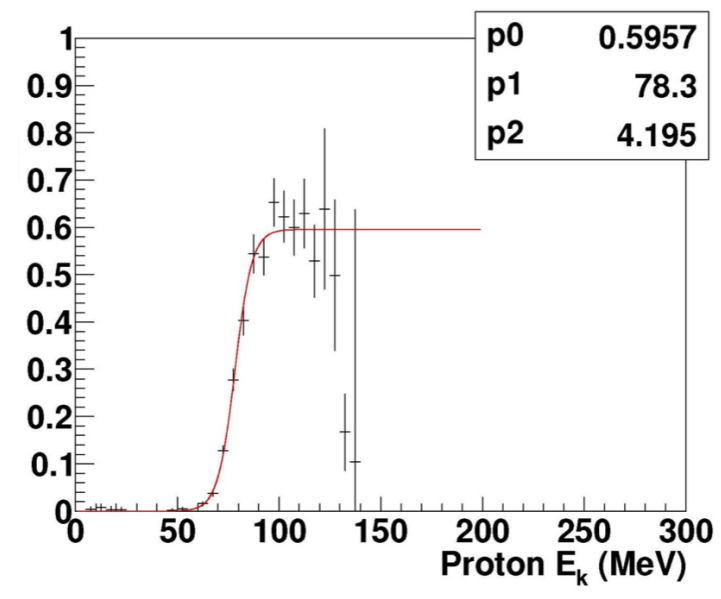

Figure 4: Proton detection efficiency in the Mainz Crystal Ball as a function of proton kinetic energy for $\pi^{0}$ photoproduction with the frozen-spin target.

\section{Meson polarizabilites}

Among important tests of low-energy QCD which are arguably unresolved by experiment, the charged and neutral pion polarizabilities are of paramount interest. [16]. For the charged pion polarizability (CPP), the constraint placed on the intrinsic even-parity sector of QCD by the polarizability is entirely complementary to the constraint placed on the intrinsic odd-parity (anamalous) sector of QCD by precision measurements of $\pi^{0}$ radiative decay [17]. The $O\left(p^{4}\right)$ prediction for the electric and magnetic polarizabilities is given by,

$$
\alpha_{\pi^{+}}=-\beta_{\pi^{+}}=\frac{4 \alpha}{m_{\pi} F_{\pi}^{2}}\left(L_{9}^{r}-L_{10}^{r}\right)
$$


The sum of low-energy constants $L_{9}^{r}+L_{10}^{r}$ is fixed by the ratio $F_{A} / F_{V}$ in charged pion radiative decay, $\pi^{+} \rightarrow e^{+} v \gamma$. This ratio has been measured by the PIBETA experiment with an error of approximately $3 \%$ [18]. The $O\left(p^{6}\right)$ corrections are predicted to be small [19] [20], with

$$
\begin{gathered}
\alpha_{\pi^{+}}-\beta_{\pi^{+}}=(5.7 \pm 1.0) \times 10^{-4} \mathrm{fm}^{3} \\
\alpha_{\pi^{+}}+\beta_{\pi^{+}}=(0.16 \pm 1.0) \times 10^{-4} \mathrm{fm}^{3}
\end{gathered}
$$

Calculations by Ramsey-Musolf and collaborators show that CPP, and more generally $\gamma^{*} \gamma^{*} \rightarrow$ $\pi^{+} \pi^{-}$with virtual photons, is an important part of the hadronic light-by-light scattering correction for muon $(g-2)_{\mu}$ [21] [22]. Marciano and collaborators have put the discrepancy between theory and experiment for $(g-2)_{\mu}$ at over the $3 \sigma$ level, [23]

$$
\Delta a_{\mu}=a_{\mu}^{E X P}-a_{\mu}^{T H}=287(83) \times 10^{-11}
$$

The Ramsey-Musolf calculations show that including CPP in the hadronic light-by-light scattering term increases the discrepancy between theory and experiment by as much as $60 \times 10^{-11}$, depending on the choice of $\alpha_{\pi^{+}}-\beta_{\pi^{+}}$and the QCD model used for calculating the $\mathrm{Q}^{2}$ dependence of the polarizability.

For the neutral pion polarizability, the polarizabilities can be calculated from one loop chiral contributions which are free of unknown parameters. The polarizabilities are expressed in terms of fundamental constants: the fine structure constant, the pion mass, and the pion decay constant:

$$
\begin{gathered}
\alpha_{\pi^{0}}+\beta_{\pi^{0}}=0 \\
\alpha_{\pi^{0}}-\beta_{\pi^{0}}=-\frac{\alpha}{48 \pi^{2} m_{\pi} F_{\pi}^{2}}=-1.1 \times 10^{-4}
\end{gathered}
$$

\subsection{Charged pion polarizability: experimental status}

There are three reaction channels that have been used to constrain CPP:

- $e^{+} e^{-} \rightarrow \gamma \gamma \rightarrow \pi^{+} \pi^{-}$. For s-wave photo-production, this reaction is sensitive to the combination $\alpha_{\pi^{+}}-\beta_{\pi^{+}}$. Experiments have yielded values of $\alpha_{\pi^{+}}-\beta_{\pi^{+}}$ranging from 4.4 to 52.6 in the standard units of $10^{-4} \mathrm{fm}^{3}$.

- Radiative pion photoproduction, $\gamma p \rightarrow \gamma^{\prime} \pi^{+} n$ at low momentum transfer to the recoil nucleon. This reaction has been investigated most recently at Mainz [24], with the result $\alpha_{\pi^{+}}-\beta_{\pi^{+}}=$ $11.6 \pm 1.5_{\text {stat }} \pm 3.0_{\text {sys }} \pm 0.5_{\text {model }}$

- Primakoff scattering of a high energy pion in the Coulomb field of a heavy nucleus, $\pi A \rightarrow$ $\pi^{\prime} \gamma A$. There have been two published results using this reaction. The older result, from Serpukov [25] , is $\alpha_{\pi^{+}}-\beta_{\pi^{+}}=13.6 \pm 2.8_{\text {stat }} \pm 2.4_{\text {sys }}$. The newest result from COMPASS at CERN [26] gives $\alpha_{\pi^{+}}-\beta_{\pi^{+}}=3.8 \pm 1.4_{\text {stat }} \pm 1.6_{\text {sys. }}$, where the constraint $\alpha_{\pi^{+}}=-\beta_{\pi^{+}}$was used in the analysis. Figure 5 shows the momentum transfer distributions for the Compass pion and muon scattering data, where the muon peak at low q is used as a line shape to extract the pion Primakoff yield. 


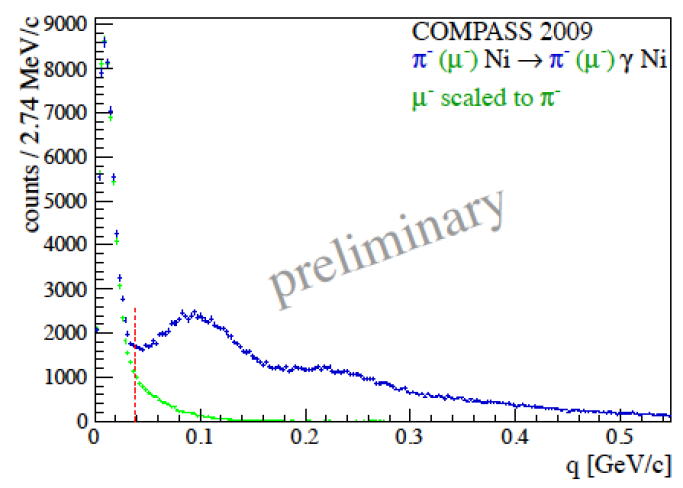

Figure 5: The Compass momentum transfer distributions for incident pion and muon beams of energy 190 $\mathrm{GeV}$ on a ${ }^{57} \mathrm{Ni}$ target.

Regarding the $\gamma \gamma \rightarrow \pi^{+} \pi^{-}$analyses [27], they were hampered by sparse data, $\approx 200$ data points in the kinematic region of interest $W<500 \mathrm{MeV}$, and by the lack of an accurate theoretical model that can predict the dependence of the total and differential cross sections on the polarizabilities. On the theoretical side, there has been considerable progress in the last decade. Pasquini and collaborators demonstrated that pion polarizability predictions from dispersion theory and ChPT are consistent [28], thereby providing a solid theoretical basis for performing calculations of $\gamma \gamma \rightarrow \pi^{+} \pi^{-}$. Pennington and collaborators performed an amplitude analysis of $\gamma \gamma \rightarrow \pi \pi$ data to study the accuracy and reliability of extracting the pion polarizability from these data [29]

The JLab Charged Pion Polarizability experiment (CPP) E12-13-008 is designed to make a precision measurement of the charged pion polarizability by means of a high statistics measurement of the $\gamma \gamma \rightarrow \pi^{+} \pi^{-}$reaction near $2 \pi$ threshold. The two-photon cross section can be accessed through the Primakoff reaction $\gamma A \rightarrow \pi^{+} \pi^{-} A$ reaction at very low momentum transfer [30] [31] [32],

$$
\frac{d^{2} \sigma}{d \Omega_{\pi \pi} d M_{\pi \pi}}=\frac{2 \alpha Z^{2}}{\pi^{2}} \frac{E_{\gamma}^{4} \beta^{2}}{M_{\pi \pi}} \frac{\sin ^{2} \theta}{Q^{4}}\left|F\left(Q^{2}\right)\right|^{2}\left(1+P_{\gamma} \cos 2 \phi_{\pi \pi}\right) \sigma(\gamma \gamma \rightarrow \pi \pi)
$$

where $\mathrm{M}_{\pi \pi}$ is the invariant mass of the $2 \pi$ system, $\mathrm{P}_{\gamma}$ is the linear polarization of the incident beam, and $\phi_{\pi \pi}$ is the azimuthal angle of the $\pi \pi$ system.

In the kinematic region of interest, $W_{\pi \pi}<0.5 \mathrm{GeV}$, cross sections for $\gamma \gamma \rightarrow \mu^{+} \mu^{-}$are approximately ten times larger than cross sections for $\gamma \gamma \rightarrow \pi^{+} \pi^{-}$[27]. To permit muon rejection at a sufficiently high level, the experiment will use a muon detection system consisting of 8 MWPCs with about $140 \mathrm{~cm}$ of iron distributed between the detectors. The iron will serve to filter out and remove charged pions, leaving only muons to track through the system. To minimize trigger bias against $\gamma A \rightarrow \pi^{=} \pi^{-} A$ signal events, the experiment will be triggered by two charged tracks in the Time-of-Flight (TOF) system.

\subsection{Neutral pion polarizability: experimental status}

In contrast to the situation for the charged pion polarizability, almost nothing is known experimentally about the neutral pion polarizability (NPP). Efforts have been made to use Crystal Ball measurements of $\gamma \gamma \rightarrow \pi^{0} \pi^{0}$ [33] to put constraints on the polarizabilities. These analyses were 
limited by the low statistics of the Crystal Ball data set, the relatively weak sensitivity of the total cross sections to the polarizabilities, and model uncertainties in calculating $\gamma \gamma \rightarrow \pi^{0} \pi^{0}$ cross sections. Because of the relatively small size of NPP relative to CPP, $\left(\alpha_{\pi^{+}}-\beta_{\pi^{+}}\right) \approx 5 \times\left(\alpha_{\pi^{0}}-\beta_{\pi^{0}}\right)$, and the absence of the Thompson term for Compton scattering on the neutral pion, which amplifies the cross section polarizability effect for the charged pion, a measurement of NPP is an experimental challenge. There are positives also: the model uncertainties in calculating $\gamma \gamma \rightarrow \pi^{0} \pi^{0}$ have largely been addressed in the recent work of Pasquini and Pennington [28] [29], and the reaction doesn't have a diffractive $\rho^{0}$ photoproduction background.

A letter-of-intent[34] is being prepared for submission to the JLab PAC to measure NPP through the $\gamma A \rightarrow \pi^{0} \pi^{0} A$ Primakoff reaction. Figure 6 shows projected statistics that can be obtained in concurrent running with the JLab CPP experiment.

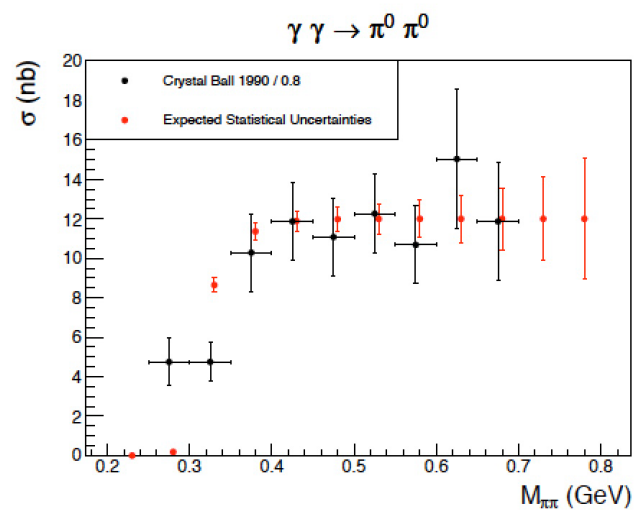

Figure 6: Cross sections and projected statistical errors for $\gamma \gamma \rightarrow \pi^{0} \pi^{0}$ The black data points are from the Crystal Ball [33]. The red data points show the projected statistics for concurrent running on $\gamma A \rightarrow \pi^{0} \pi^{0} A$ with the JLab CPP experiment .

\section{Summary and acknowledgements}

High intensity polarized photon beams and polarized targets, both"standard" and "active" frozen-spin targets, are key elements needed to reduce uncertainties in the proton polarizabilities below \pm 0.4 in the standard units. Further effort is needed on neutron polarizabilities, and the active He target development at Mainz is promising. Measurements of the charged and neutral pion polarizabilties requires multi-GeV photon/hadron beams, and precision measurement technique. Experiments are being planned for JLab and Compass.

The author acknowledges and thanks M. Biroth, C. Collicott, H. Grei $\beta$ hammer, D. Hornidge, D. Lawrence, and P. Martel for their assistance in the preparation of this talk.

\section{References}

[1] F.J. Federspiel, et al., Phys. Rev. Lett. 67 (1991) 1511.

[2] J.A. McGovern, D.R. Phillips, H.W. Gießhammer, Eur. Phys. J. A 49 (2013) 12. 
[3] V. Sokhoyan et al., Eur. Phys. J. A (2017) 53: 14

[4] L.S. Myers, et al., Phys. Rev. Lett. 113 (2014) 262506.

[5] J. Annand, proposal to the Mainz Microtron MAMI-A2, (2013).

[6] B. Pasquini, P. Pedroni, D. Drechsel, Phys. Lett. B 687 (2010) 160.

[7] M. Camen, et al., Phys. Rev C 65 (2002) 032202.

[8] B. Pasquini, D. Drechsel, M. Vanderhaeghen, Phys. Rev. C 76 (2007) 015204.

[9] G. Blanpied, et al. Phys. Rev C 64 (2001) 025203.

[10] C. Collicott, (Ph.D. thesis), Dalhousie University, Halifax, NS, (2015).

[11] P.Martel, et al. and the Mainz A2 collaboration, Phys. Rev. Lett. 114, 112501 (2015).

[12] V. Lensky, V. Pascalutsa, Eur. Phys. J C 65( 210) 195.

[13] H. Grießhammer, J. McGovern, D. Phillips, G. Feldman, Prog. Part. Nucl. Phys. 67 (2012) 841.

[14] D. Paudyal, PhD thesis, U. Regina (2018).

[15] Private communication, M. Biroth (2018).

[16] B. Holstein, S. Scherer, arXiv:1401.0140v1 [hep-ph], (2014).

[17] R. Miskimen, Annual Review of Nuclear and Particle Science 61, 1, (2011).

[18] M. Bychkov et al., Phys. Rev. Lett. 103, 051802, (2009).

[19] U. Burgi, Nuclear Physics B479, 392, (1996).

[20] J. Gasser, M.A. Ivanov, M.E. Sainio, Nuclear Physics B745, 84, (2006).

[21] K. Engel, H. Patel, M. Ramsey-Musolf, Phys. Rev. D 86, 037502, (2012).

[22] K. Engel, M. Ramsey-Musolf, arXiv:1309.2225 [hep-ph], (2013).

[23] M. Passera, W.J. Marciano, A. Sirlin, arXiv:1001.4528 [hep-ph], (2010).

[24] J. Ahrens et al., Eur. Phys. J. A23, 113, (2005).

[25] Yu.M. Antipov et al., Phys. Lett. B121, 445 (1983).

[26] C. Adolph, et al., Phys. Rev. Lett. 114, 062002 (2015).

[27] J. Boyer et al. Mark-II collaboration, Phys. Rev. D42, 1350 (1990).

[28] B. Paquini, D. Drechsel, and S. Scherer, Phys. Rev. C 77, 06521 (2008).

[29] Ling-Yun Dai, M. R. Pennington, Phys. Rev. D 94, 116021, (2016)

[30] A. Halprin, C. Anderson, H. Primakoff, Phys. Rev. 152, 1295 (1966).

[31] L. Stodolsky, Phys. Rev. Lett. 26, 404 (1971).

[32] V. Budnev, I. Ginzburg, G. Meledin, V. Serbo, Phys. Rep. 15, No. 4, 181 (1975),

[33] H. Marsiske et al., Phys. Rev. D41:3324, 1990.

[34] Private communication, M. Ito, J. Goity, D. Lawrence, E.S. Smith, B. Zihlmann, R. Miskimen, I. Larin, and A. Austregesilo (2019). 